\title{
Etude de la germination de cinq provenances de Tamarindusindica L. en conditions de stress hydrique au Sénégal
}

\author{
Cheikh Oumar SAMB ${ }^{1 *}$, Marème NIANG ${ }^{2}$, Arona Ndiaye Samba SAMBA ${ }^{3}$, \\ Mbaye Ndoye SALL ${ }^{2}$, Ndiaga CISSE ${ }^{2}$, Macoumba DIOUF ${ }^{2}$ et Patrick VAN DAMME ${ }^{4,5,6}$ \\ ${ }^{I}$ Centre National de Recherches Forestières (ISRA/CNRF) BP 2312 Dakar, Sénégal. \\ ${ }^{2}$ Centre d'Etudes Régional pour l'Amélioration à l'Adaptation à la Sécheresse \\ (ISRA / CERAAS) BP 3320, Thiès, Sénégal. \\ ${ }^{3}$ Ecole Nationale Supérieure d'Agriculture (ENSA,Université de Thiès) BPA 296 Thiès, Sénégal. \\ ${ }^{4}$ Ghent University, Laboratory of Tropical and Subtropical Agriculture and Ethnobotany (LTSAE), Coupure \\ links 653, 9000 Gent, Belgium. \\ ${ }^{5}$ World Agroforestry Centre, United Nations Avenue, Gigiri, P.O. Box 30677, Nairobi, 00100, Kenya. \\ ${ }^{6}$ Faculty of Tropical Agrisciences, Czech University of Life Sciences Prague, Kamycka 129, Prague 6 - \\ Suchdol, 165 21, Czech Republic. \\ *Auteur correspondant ; E-mail : omarsamb2004@yahoo.fr ; Tel : 221775168490
}

\section{RESUME}

Ce présent travail se propose d'évaluer le comportement germinatif de cinq provenances de tamarinier (Tamarindus indica L.) dans des conditions de stress hydrique. Six concentrations croissantes 0 ; - 1,4 ; - 2,8 ; 4,$2 ;-5,6 ;-7$ bar de polyéthylène glycol (PEG 6000) ont été testées. Les résultats ont montré une variabilité de la germination de l'espèce en fonction de l'intensité du stress et de la provenance. Le seuil critique de germination des provenances du tamarinier est de -7 bar. La provenance Sakkal de la zone sahélienne s'est montrée plus tolérante au stress hydrique avec un taux de germination de $2 \%$ en conditions de stress sévère. Toutefois, ces faibles résultats sont imputables aux conditions de conservation des graines. Ces informations sur l'écologie et la physiologie de la germination du tamarinier sont vitales, non seulement pour comprendre les variations génétiques des différentes populations de tamarinier au Sénégal, mais aussi pour développer des stratégies pour la conservation de la biodiversité et la restauration des forêts tropicales.

(C) 2015 International Formulae Group. All rights reserved.

Mots clés : Germination, provenances, Tamarindus indica L., stress hydrique, Sénégal.

\section{INTRODUCTION}

Au Sénégal, une réduction considérable $\mathrm{du}$ couvert ligneux ces trois dernières décennies, associée à une érosion de la diversité biologique (CSE, 2003), a été constatée dans l'ensemble des écosystèmes naturels. Cette dégradation de la couverture végétale est attribuée à l'avancée des fronts agricoles, à leur surexploitation, à leur faible régénération (Ouedraogo, 2008; Traoré, 2008; Agali, 2009; Belem, 2009) et aux changements climatiques (sécheresse) (Bonkoungou et al., 2002; Muok et Alem, 2011). Ce qui entraîne une situation 
défavorable pour l'épanouissement de la végétation (Botoni et Reij, 2009). D'où la nécessité de réhabiliter et de restaurer la strate arbustive et arborée par l'introduction d'espèces forestières autochtones comme le tamarinier, espèce très menacée (Bourou, 2012) dans un contexte de changement climatique. Cependant, cette restauration doit passer par une évaluation des capacités germinatives de l'espèce en conditions de stress hydrique afin de déterminer le seuil critique de germination et d'identifier les provenances de tamarinier les plus tolérantes aux contraintes hydriques.

L'objectif de ce travail est d'évaluer le comportement germinatif de cinq provenances de tamarinier en conditions de stress hydrique. Notre hypothèse d'étude est que les différentes provenances présenteraient une différenciation par leur aptitude à résister au stress hydrique au stade de germination.

\section{MATERIEL ET METHODES Matériel}

Le matériel végétal est constitué des graines de $T$. indica L. collectées en 2010 dans cinq sites différents suivant le gradient pluviométrique nord-sud du Sénégal. Il s'agit de Sakkal (zone sahélienne), de Gnibi, Mbassis, Dogoro (zone soudano-sahélienne) et de Vélingara (zone soudanienne) (Figure 1). Elles ont été traitées puis conservées à l'air ambiant au Centre d'Etudes Régional pour l'Amélioration à l'Adaptation à la Sécheresse (CERAAS) de Thiès.

\section{Méthodes}

Les graines ont été trempées dans de l'acide sulfurique $96 \%$ pendant $10 \mathrm{mn}$ afin de lever l'inhibition tégumentaire. Le test de germination a été effectué sous différentes concentrations de polyéthylène glycol (PEG 6000). L'essai porte sur 80 graines, soit quatre répétitions de 20 graines par boîte de pétri. Elles sont ensuite mises à germer dans des boites de pétri de diamètre $8 \mathrm{~cm}$ tapissées de papier filtre. Dans chaque boîte de pétri sont versés $10 \mathrm{ml}$ d'eau distillée et $10 \mathrm{ml}$ de solutions contenant $-1,4 ;-2,8 ;-4,2 ;-5,6$ et 7 bar. La quantité de PEG 6000 utilisée est calculée en fonction de l'équation de Michel et Kauffman (1973). Les boites de pétri sont ensuite placées dans une chambre de culture à une température de $27 \pm 2{ }^{\circ} \mathrm{C}$ et à l'obscurité pour une période de 25 jours. Selon Danthu et al. (2003), la germination est indifférente à la lumière. Un suivi quotidien a été fait pour estimer la date de germination des différentes provenances. Une graine est considérée comme germée lorsque la radicule a percé les téguments et atteint $2 \mathrm{~mm}$ de long (Côme, 1970).

Les variables mesurées ont porté sur le taux de germination finale (TGF), le délai de germination (DG) et la cinétique d'évolution de germination (CEG). Les données collectées ont été soumises à une analyse de variance avec le logiciel Statistix 8.1 puis à une comparaison des moyennes à l'aide du test de Least Significant Difference (LSD) au seuil de $5 \%$.

\section{RESULTATS}

\section{Taux de germination}

Les résultats des taux de germination finale des graines des différentes provenances de tamarinier étudiées sont présentés dans la Figure 2. L'analyse de la Figure 2 montre que la germination varie en fonction de la concentration du PEG et de la provenance. Les taux de germination diminuent significativement avec l'augmentation de la concentration du PEG pour toutes les provenances. L'espèce semblait être sensible au stress hydrique. Cette sensibilité se traduit par l'absence de germination à la pression osmotique de -7 bar. Toutefois, il est important de noter à une pression osmotique de -5,6 bar, la capacité germinative des provenances Dogoro, Gnibi, Mbassis et Vélingara est significativement affectée. Leur pourcentage de germination a fortement diminué comparé à la pression hydrique de 0 bar (absence de stress) et a passé de 31 à $0 \%$ pour Dogoro, 25,4 à $0 \%$ pour Mbassis, 19,41 à $0 \%$ pour Gnibi, 10 à $2 \%$ pour Sakkal et 35 à $0 \%$ pour Vélingara. Donc, la provenance Sakkal semblait être plus résistante à une pression de $-5,6$ bar (Figure 2). 


\section{Délai de germination}

L'analyse du Tableau 1 montre que le stress hydrique affecte le délai de germination. On remarque à l'absence de stress hydrique, le délai de germination du tamarinier varie de 2 à 4 jours (Tableau 1). Par contre, on constate une augmentation considérable du temps moyen de germination quand la concentration de PEG devient plus sévère. Toutefois, la provenance Vélingara enregistre les meilleurs délais de germination pour des pressions osmotiques inférieures à $-5,6$ bar (Tableau 1).Cependant, la provenance Sakkal enregistre un délai de 14 jours au potentiel osmotique de $-5,6$ bar. L'effet du stress hydrique se traduit par une augmentation du temps de germination de l'espèce.

\section{Cinétique d'évolution de germination}

La Figure 3 présente l'évolution de la germination de cinq provenances de tamarinier en fonction du temps et des niveaux de stress. L'analyse de la Figure 3 montre que l'allure générale des courbes de cinétique d'évolution de la germination est similaire pour toutes les provenances étudiées à tous les niveaux de pression osmotique, sauf à une pression de $-5,6$ bar. Toutefois, la courbe de la provenance Vélingara se situe audessus de celles des autres provenances (Dogoro, Mbassis, Gnibi, Sakkal) pour les concentrations $0 ;-1,4 ;-2,8 ;-4,2$ bar. Alors, à une pression de $-5,6$ bar la germination de Vélingara, Mbassis, Gnibi et Dogoro a été annulée.

Ces courbes d'évolution de germination affichent trois phases :

$>\quad$ Une première phase marquée par un faible taux de germination avec une durée variable selon la concentration de PEG 6000 ; $>\quad$ Une deuxième phase marquée par une augmentation du taux de germination ;

$>\quad$ Et une troisième phase stationnaire traduisant la capacité germinative des provenances en fonction des différentes concentrations de PEG 6000.

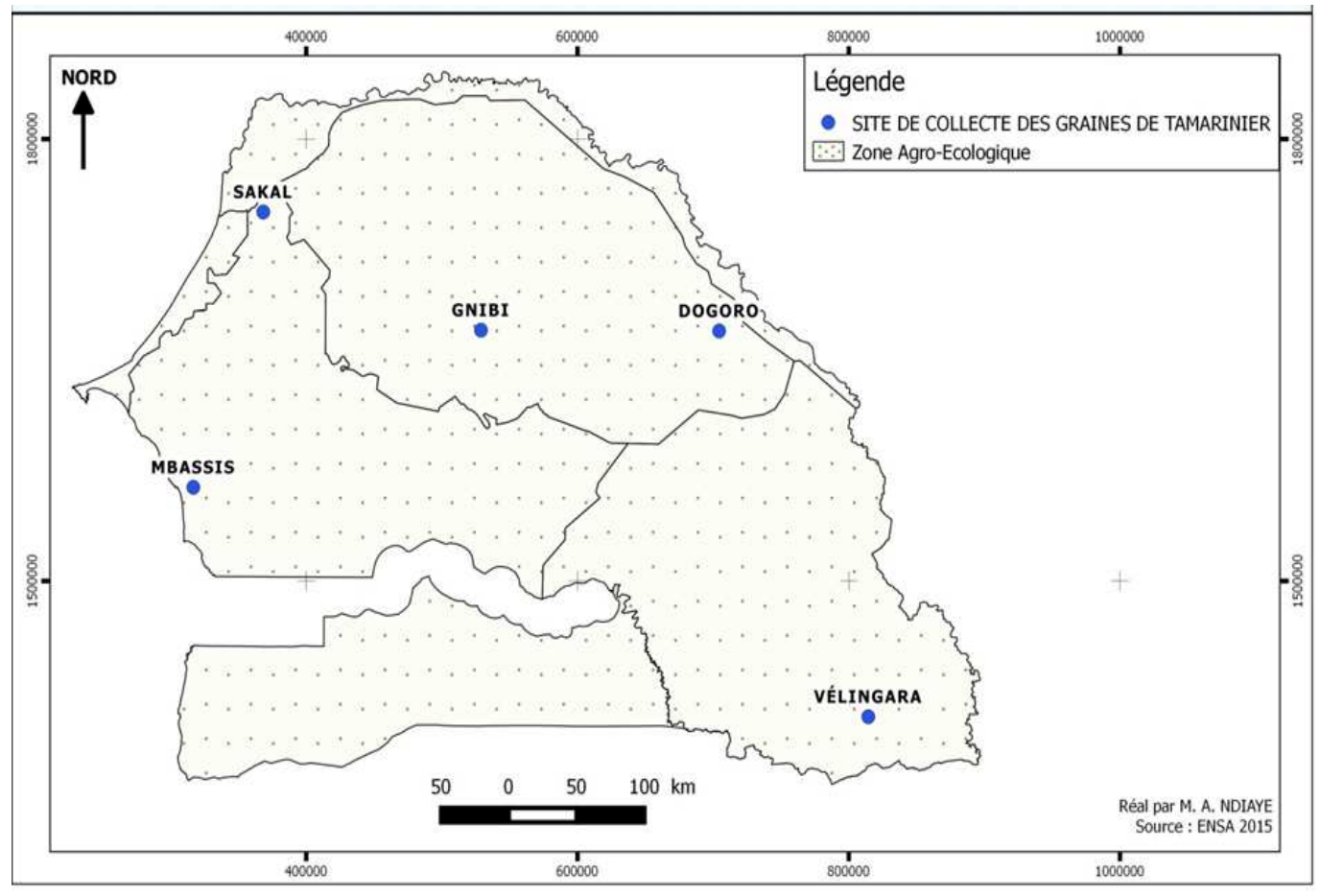

Figure 1 : Sites de collecte des graines de $T$. indica $\mathrm{L}$. 


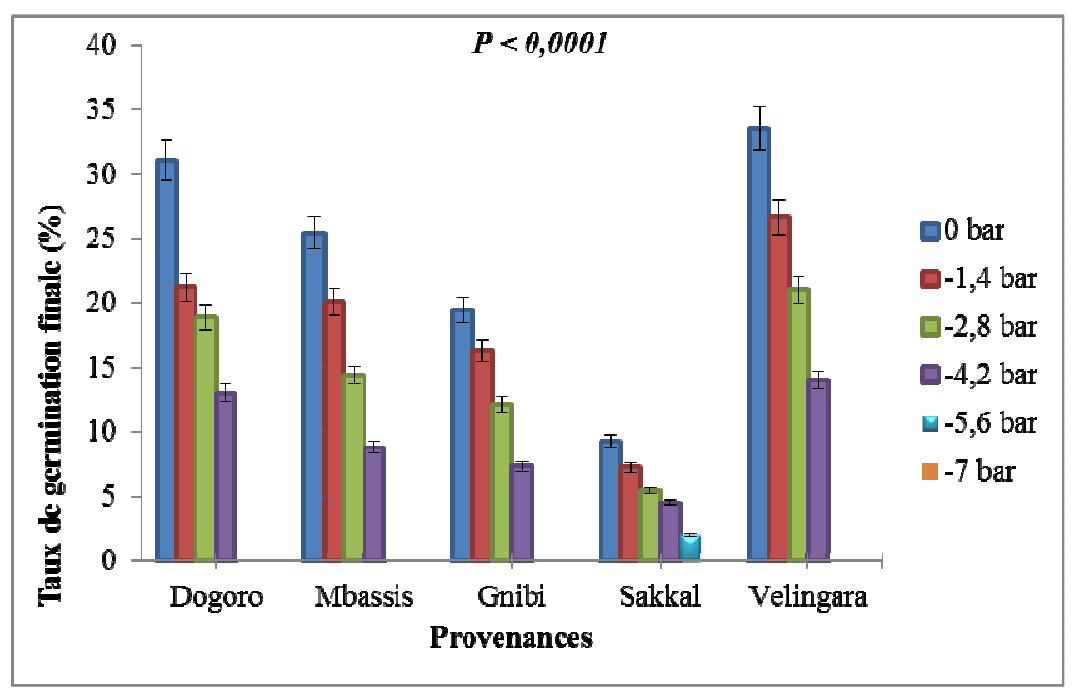

Figure 2 : Variation du taux de germination finale des provenances de $T$. indica $L$. en fonction de la pression osmotique de la solution de PEG.

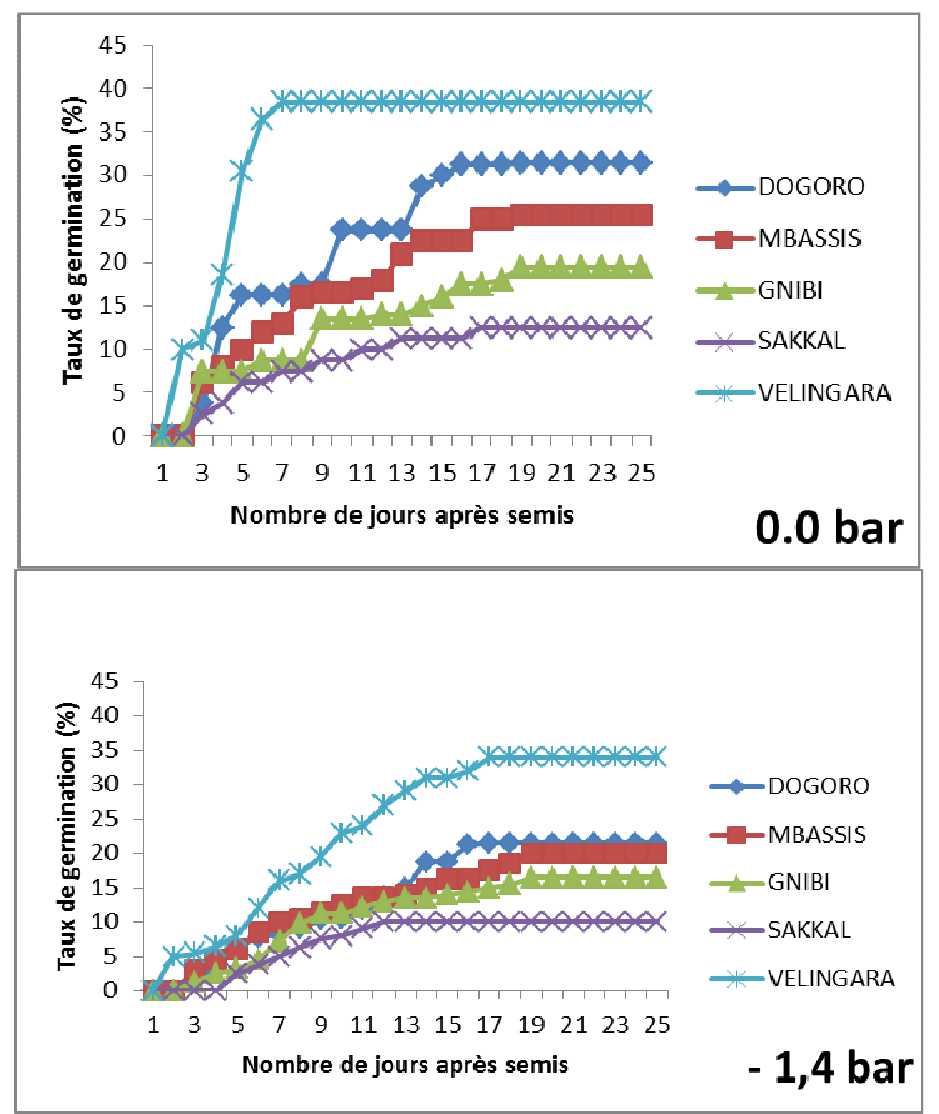




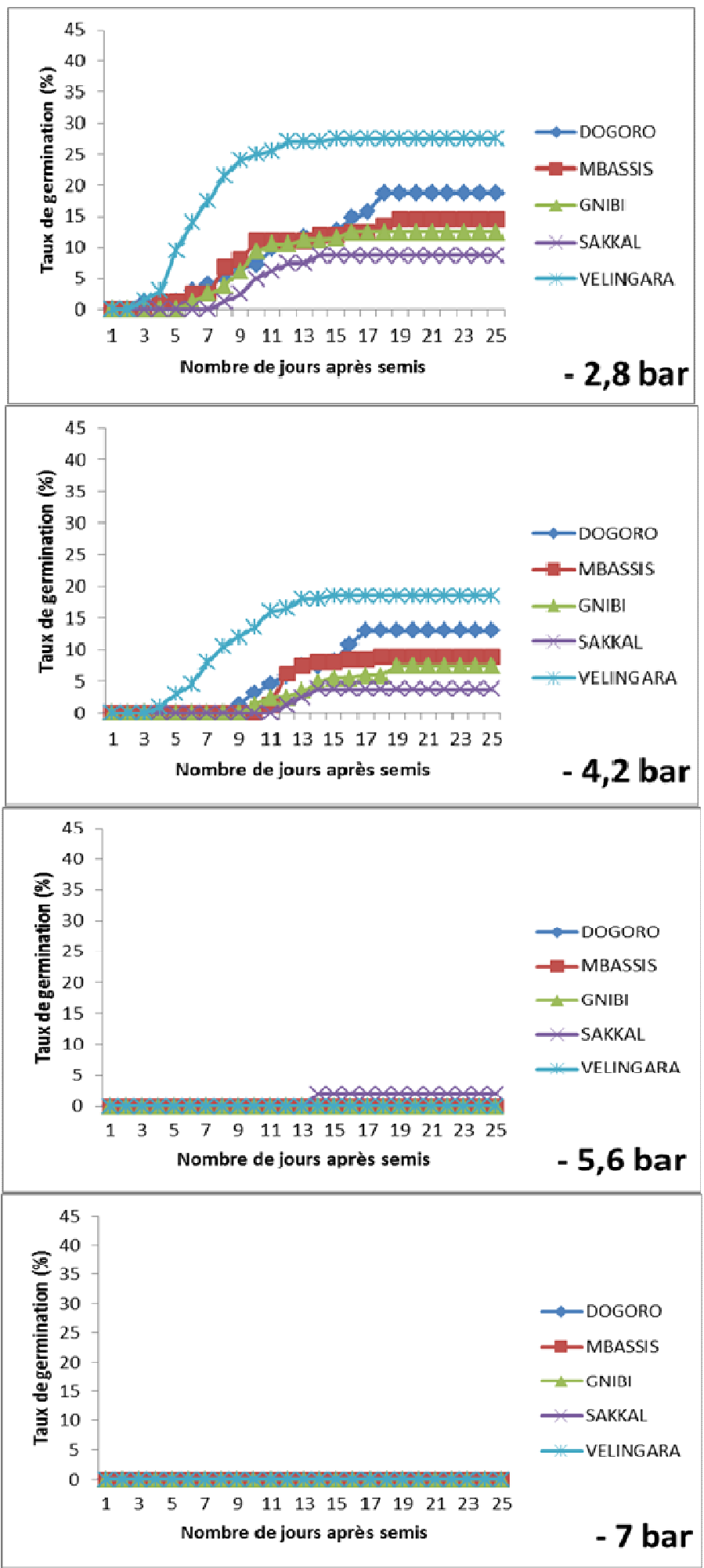

Figure 3 : Cinétique d'évolution de la germination des cinq provenances de $T$. indica $\mathrm{L}$. 
Tableau 1: Variation du délai de germination (jours) des provenances de T. indica L. en fonction du potentiel osmotique (bar).

\begin{tabular}{lcccccc}
\hline \multicolumn{7}{c}{ Potentiel osmotique (bar) } \\
\hline provenances & Témoin (0) & $\mathbf{- 1 , 4}$ & $\mathbf{- 2 , 8}$ & $\mathbf{- 4 , 2}$ & $\mathbf{- 5 , 6}$ & $\mathbf{- 7}$ \\
Dogoro & 3,5 & 4,25 & 6 & 10,25 & 0 & 0 \\
Mbassis & 3,25 & 3,75 & 7 & 11,75 & 0 & 0 \\
Gnibi & 3 & 4,25 & 7,25 & 11,25 & 0 & 0 \\
Sakkal & 3,75 & 5,75 & 8,75 & 9,75 & 14 & 0 \\
Vélingara & 2,5 & 3,75 & 4 & 7,25 & 0 & 0 \\
\hline
\end{tabular}

\section{DISCUSSION}

Nos résultats montrent que l'espèce $T$. indica $\mathrm{L}$. est sensible au stress hydrique. Le seuil critique de germination est de -7 bar. Plus la pression osmotique est importante, plus le taux de germination diminue. Nos résultats confirment les travaux de Neffati et Akrimi (1996) qui indiquent que la germination de Accacia raddiana s'annule pratiquement à -7 bar. Cependant, les travaux de recherches (Gouzis et al., 2003) indiquent que lorsque les graines de Acacia tortilis et Acacia senegal sont soumises à une contrainte hydrique simulée par adjonction de polyéthylène glycol (PEG 6000) à l'eau d'imbibition, leur germination n'est pas significativement inférieure à celles des témoins (absence de PEG) que pour un potentiel hydrique inférieur à $-1,8 \mathrm{MPa}$. Si la pression est égale à $-2,1 \mathrm{MPa}$, un quart environ des graines germent encore. La valeur limite de germination de l'espèce est de -7 bar.

Le stress hydrique diminue le taux de germination et allonge le délai de germination du tamarinier. Ce résultat corrobore ceux de Hardegre et Emmerich (1994), de Ferchichi (2004) sur Cenchrus ciliatus et de Jaoudi et al. (2010) sur Acacia tortilis. Le retard de germination peut être expliqué aussi par le temps nécessaire à la graine pour mettre en place des mécanismes qui lui permettent d'ajuster son potentiel osmotique interne par rapport au milieu (Bliss et al., 1986 ; Jaoudi et al., 2011).
Nos résultats montrent également l'existence d'une différence de sensibilité entre les cinq provenances. La provenance Sakkal de la zone sahélienne semble être plus tolérante au stress hydrique vue que la diminution du taux de germination n'est pas drastique comme chez la provenance Vélingara de la zone soudanienne. Cette performance de Sakkal s'expliquerait par son appartenance géographique d'une zone aride à faible pluviosité. Donc, cette provenance habituée aux conditions sèches supporterait mieux l'effet du stress osmotique (Roach et al., 1987). Dirik (2000) indique qu'il y a des relations entre les facultés à germer des provenances de Cèdre du Liban (Cedrus Libani A. Rich.) face au stress hydrique et les conditions écologiques de leurs milieux naturels. Cependant, il est difficile de relier la tolérance aux contraintes hydriques, au moment de la germination, à l'écologie de l'espèce même (Le Floc'h et al., 1989 ; Jaoudi et al., 2010).Les travaux de recherches de Sharma et al. (1973) sur cinq espèces pastorales, de Ndour et al. (1998) sur quelques acacias africains, de Sy et al. (2001) sur sept espèces légumineuses sahéliennes, de Jaoudi et al. (2010) sur Acacia tortilis ont démontré que les espèces les plus tolérantes au stress hydrique au cours de la germination ne sont pas nécessairement celles qui sont les plus adaptées à la sécheresse au stade adulte. 


\section{Conclusion}

Notre étude a montré que le stress hydrique a un effet dépressif sur la germination du tamarinier. Les résultats ont fait ressortir que la germination du tamarinier est affectée à -7 bar. Les provenances Mbassis, Gnibi, Dogoro, Vélingra sont plus sensibles au stress hydrique et celle de Sakkal semblerait être plus tolérante et pourrait être utile pour la domestication de l'espèce. Toutefois, la provenance Vélingara est remarquable avec un TGF et un DG plus intéressants. Donc, ces paramètres (TGF, DG) pourraient être considérés comme critères précoces de sélection des espèces forestières tolérantes au stress hydrique.

\section{REMERCIEMENTS}

Cette étude a bénéficié de l'appui du Projet DADOBAT pour la fourniture des graines. Les auteurs en remercient le Coordonnateur de ce projet, l'ISRA, l'ENSA et particulièrement le Centre d'Etudes Régional pour l'Amélioration à l'Adaptation à la Sécheresse (ISRA / CERAAS) de Thiès pour l'appui en logistique et tout le personnel du Laboratoire pour leur collaboration.

\section{REFERENCES}

Agali AB. 2009. Diversité, structure et perceptions locales des espèces ligneuses fourragères dans le terroir de Torodi, Ouest Niger. Mémoire de DEA en Biologie et Ecologie végétales : Université d'Ouagadougou, Burkina Faso, 48p.

Almansoury M, Kinet JM, Lutts S. 2001. Effect of salt and osmotic stresses on germination in durum wheat (Triticum durum Desf). Plant and soil, 231: 243255.

Bayarassou Z. 2011. Effet du stress salin sur la germination de l'Atriplex canescens et de l'Atriplex halimus, 49p.
Belem B. 2009. Ethnobotanique et conservation de Bombax costatum PEL. Et VUIL. (Faux Kapokier) dans les systèmes de production agricole dans le plateau central, Burkina Faso. Thèse de Doctorat unique en sciences biologiques appliquées : Université de Ouagadougou, Burkina Faso, 143p.

Bliss RD, Patt-Alliola KA, Thomsin W. 1986. The inhibitory effect of $\mathrm{NaCl}$ on barley germination. Plant cell Environ., 9: 727733.

Bonkoungou GE, Djimde M, Ayuk ET, Zoungrana I, Tchoundjeu Z, Niang A, Ndiaye S, Bernard OM, Alem S. 2011. Tamarindusindica,

Tamarinier. Conservation et Utilisation Durable des Ressources Génétiques des Espèces Ligneuses Alimentaires Prioritaires de l'Afrique Subsaharienne. Biodiversity International: Rome, Italie; 8.

Botoni E, Reij C. 2009. Silent transformation of environment and production system in the Sahel: impact of public and private investments in natural resource management. CILSS et Université libre d'Amsterdam.

Bourou S, Ndiaye F, Diouf M, Van Damme P. 2011. Effet de l'inoculation mychorizienne sur des écotypes du tamarinier. Journal of Applied Biosciences, 46 : 3093-3102.

Bourou S. 2012. Étude éco physiologique du Tamarinier (Tamarindusindica L.) en milieu tropical aride. Thèse de Docteur en Sciences Biologiques Appliquées : section Agronomie: Faculty of Bioscience Engineering : Universiteit Gent, 193p.

Come D. 1970. Les Obstacles à la Germination. Ed. Masson et Cie: Paris, $162 \mathrm{p}$.

CSE / LADA. 2003. Evaluation de la Dégradation des Terres au Sénégal. CSE : Dakar ; 62. 
Dantu P, Roussel J, Gaye A, El Mazzoudi EH. 1996. Baobab (Adansoniadigitata L.) seed pretreatments for germination improvement. Seed science and Technology, 23(2): 469-475.

Dirik H. 2000. Effet du stress hydrique osmotique sur la germination des graines chez les provenances de Cédre du Liban (Cedrus libani A. Rich) d'origine Turque. Ann . Forest sci., 57: 367-371.

Djibril S, Mohamed OK, Diaga D, Diouf D, Ablaye DF, Sagna M, Borgel A. 2005. Growth and development of date palm (Phoenix dactylifera L.) seedlings under drought and salinity stresses. Afr. J. Biotech., 4(9): 968-972.

FAO. 1982. Espèces fruitières forestières, fiches techniques, FAO, 197p.

Gouzis M, Le Floc'h. 2003. Un Arbre au Désert Acacia raddiana. IRD Editions: Paris; 313p.

Hardeger SP, Emmerich WE. 1994. Seed germination response to polyethylene glucol (PEG 6000) solution depth. Seed. Sci \& Technol., 22: 1-7

Jaouadi W, Hamrouni L, Souayeh N, Khoudia ML. 2010. Etude de la germination des graines de Acaciatortilis sous différentes contraintes abiotiques. Base, 14(4): 643652.

Jaoudi W, Hamrouni L, Hanana M, Khouja ML. 2011. Analyse de la capacité germinative de quelques espèces de Acacia exotique.

Kẹpczyński J. 1986. Inhibition of Amaranthuscaudatus seed germination by polyethylene glycol-6000 and abscisic acid and its reversal by ethephon or 1aminocyclopropane-1-carboxylic acid. Physiologia Plantarum, 67: 588-591.

Kouakou TH, Koné M, Koné D, KouadioYJ, Zouzou M. 2008. Réponse physiologique au stade juvenile du génotype R495-2000 de cotonier (Gossypiumhirsutum L.) au déficit hydrique induit par le polyéthylène glycol. Article original. Sciences et Nature, 5(1): 81-87.

Le Floc'h E, Schoenenbereger A, Nabli MA, Valdeyron G. 1989. Biologie et écologie des principaux taxons. In Essai de Synthèse sur la Végétation et la PhytoEcologie Tunisienne : I. Éléments de Botanique et de Phytoécologie, Nabli MA (ed). Tunis: Faculté des Sciences, 51-193.

Malaki A, Ouedrago JS, Yossi H. 2002. L'agroforesterie, un outil performant pour la gestion des ressources naturelles et la lutte contre la désertification au Sahel: Bilan de dix années d'expérience en recherches - développement et perspectives. CILSS-INSAH ; ICRAF SALWA. Les monographies sahéliennes $\mathrm{N}^{\circ} 11,88 \mathrm{p}$

Michel, Kauffman. 1973. The osmotic potential of polyethylene glycol 6000 . Plant Physiol., 51(5): 914-916.

Mohamdi MO, Bouya D, Salem AOM. 2011. Etude de l'effet du stress salin $(\mathrm{NaCl})$ chez deux variétés de Tomate (Campbell 33 et Mongal). Université de Nouakchott: Facultés des sciences et techniques, Laboratoire de biotechnologies, 11p.

Moulay BN. 2011. Etude du stress hydrique sur la germination de quelques graines d'Atriplex, 48p.

Mrani Alaoui M, El Jourmi L, Ouarzane A, Lazar S, El Antri S, Zahouily M, Hmyene A. 2013. Effet du stress salin sur la germination et la croissance de six variétés marocaines de blé. J. Mater. Environ. Sci., 4(6) : 997-1004.

Ndiaye A, Faye E, Touré MA. 2014. Effet du stress salin sur la germination des grains de Gossypiumhirstum. Journal of Applied Biosciences, 80: 7081- 7092.

Ndour P, Danthu P. 1998. Effet des contraintes hydriques et salines sur la germination de quelques acacias africains. In Colloques et Séminaires : l'Acacia au 
Sénégal, Campa C, Grignon C, Gueye M, Hamon S (eds). Orstom : Paris ; 105-122.

Neffati, Akrimi. 1996. Etudes des caractéristiques germinatives des semences de quelques légumineuses spontanées de la Tunisie steppique. Actes de séminaire international. Revues des Régions Arides. 272 - 287.

Ouedraogo I. 2008. Diversité des espèces ligneuses utiles de la région nord du Burkina Faso; état des peuplements de cinq espèces d'importance socioéconomique. Mémoire d'Ingénieur en Eaux et Forêts: Université de Bobo-D, Burkina Faso, 68p.

Sharma ML. 1973. Simulation of drought and its effect on germination of five pasture species. Agron. J., 65: 982-987.
Singh C, Khajuria HN, Singh A, Sharma F. 1991. Acacias for arid regions. Acta Bot. Indica, 19: 29-32.

Sy A, Grouzis M, Danthu P. 2001. Seed germination of seven Sahelian leguminous species. J. Arid Environ., 49: 875-882.

Traoré L. 2008. Inventaire des espèces ligneuses utilitaires de la région SudOuest du Burkina Faso et état des populations de trois espèces à haute valeur économique. Mémoire de DEA en sciences Biologiques Appliquées: Université de Ouagadougou, Burkina Faso, 46p. 